\title{
Anosmia and Dysgeusia in the Absence of Other Respiratory Diseases: Should COVID-19 Infection Be Considered?
}

\author{
Noel Lorenzo Villalba ${ }^{1}$, Yasmine Maouche ${ }^{1}$, Maria Belen Alonso Ortiz ${ }^{2}$, Zaida Cordoba Sosa ${ }^{3}$, \\ Jean Baptiste Chahbazian ${ }^{1}$, Aneska Syrovatkova ${ }^{1}$, Pierre Pertoldi ${ }^{1}$, Emmanuel Andres ${ }^{1}$, Abrar-Ahmad Zulfiqar ${ }^{1}$ \\ ${ }^{1}$ Service de Médecine Interne, Diabète et Maladies Métaboliques, Hôpitaux Universitaires de Strasbourg, France \\ ${ }^{2}$ Internal Medicine Department, Dr Negrin University Hospital, Gran Canaria, Spain \\ ${ }^{2}$ Internal Medicine Department, Fuerteventura General Hospital, Fuerteventura, Spain
}

Received: 01/04/2020

Accepted: 01/04/2020

Published: 03/04/2020

\begin{abstract}
How to cite this article: Lorenzo Villalba N, Maouche Y, Alonso Ortiz MB, Cordoba Sosa Z, Chahbazian JB, Syrovatkova A, Pertoldi P, Andres E, Zulfiqar AA. Anosmia and dysguesia in the absence of other respiratory disease: should COVID-19 infection be considered? EJCRIM 2020;7: doi:10.12890/2020_001641.
\end{abstract}

Conflicts of Interests: The Authors declare that there are no competing interests.

This article is licensed under a Commons Attribution Non-Commercial 4.0 License

\section{ABSTRACT}

We describe two elderly patients evaluated at emergency departments for anosmia/dysgeusia in the absence of any other respiratory symptoms prior to or upon admission. In the current epidemiological context, clinical and biological work-up led to a diagnosis of COVID-19 infection. Unfortunately, one of the patients died during hospitalization, but the other recovered and was discharged.

\section{LEARNING POINTS}

- In the current epidemiological situation, anosmia and dysgeusia in the absence of other respiratory conditions should be carefully evaluated.

- Special attention should be given to patients with non-classic COVID-19 symptoms in order to reduce transmission and protect health providers

\section{KEYWORDS}

COVID-19, SARS-CoV-2, 2019-nCoV, anosmia, dysgeusia

\section{INTRODUCTION}

The Coronaviruses are a large family of viruses which cause illnesses ranging from the common cold to more severe disease. SARS-CoV-2 is a novel Coronavirus which was first detected in China and is now spreading globally ${ }^{[1]}$. The beginning of the COVID-19 outbreak was associated with a seafood market selling live animals which most patients had attended. However, with progression of the outbreak, the illness is now spread by person-to-person transmission. The common serious manifestation of COVID-19 infection is pneumonia.

\section{CASE DESCRIPTION}

\section{Case 1}

An 85-year-old man was admitted to the emergency department for a 4-day history of sudden anosmia and fatigue without any history of fever, cough or shortness of breath. He had previously refused to visit his primary care doctor. His medical history was relevant for hypertension, ischaemic heart disease and type 2 diabetes. He lived with his wife and there was apparently no close contact with anybody with acute respiratory symptoms. Upon admission, the patient was afebrile, his blood pressure was $110 / 80 \mathrm{mmHg}$, heart rate was $82 \mathrm{bmp}$ and oxygen saturation was $94 \%$ on room air. On physical examination, he was alert but asthenic. 
Heart sounds were regular without murmurs, but vesicular breath sounds were bilaterally decreased in both pulmonary bases on auscultation. Blood tests showed elevated C-reactive protein $(210 \mathrm{mg} / \mathrm{l})$, lymphopenia and thrombopenia ( $\left.88 \times 10^{\circ} / \mathrm{I}\right)$. Electrolytes and liver function tests were within normal ranges and the glomerular filtration rate (GFR) was $65 \mathrm{ml} / \mathrm{min}$. D-dimer was elevated (3,500 ng/ml). A thoracic angiogram was normal. PCR for SARS-CoV-2 came back positive, confirming the diagnosis of COVID-19. The patient was treated in a normal hospital bed. Three days after hospital admission (6 days after symptom onset), the patient developed acute respiratory distress syndrome (ARDS) and died.

Case 2

An 80-year-old woman was admitted to the emergency department for a 5-day history of taste loss preceding smelling problems and fatigue. The was no history of fever, chills or dyspnoea before her admission. Her medical history was relevant for hypertension, heart failure and end-stage chronic renal disease. She lives with her daughter who works in a nursing home and is apparently in good health. Upon admission, the patient was febrile $\left(38.1^{\circ} \mathrm{C}\right)$, and had a blood pressure of $100 / 50 \mathrm{mmHg}$, heart rate of $90 \mathrm{bmp}$ and oxygen saturation of $92 \%$ on room air. On physical examination, she was alert and oriented to time, space and person. Heart sounds were regular without murmurs but the vesicular breath sounds were decreased in the right pulmonary base on auscultation. Blood tests showed leucocytosis (12.6 $\left.\times 10^{\circ} / \mathrm{l}\right)$ with a predominance of polynuclear neutrophils $\left(11.72 \times 10^{9} / \mathrm{I}\right)$ but normal lymphocyte and platelet counts. Electrolytes, liver, renal and coagulation tests were within normal ranges. The chest CT demonstrated the presence of ground-glass opacification in the right lower lobe. The investigations were completed with PCR for SARS-CoV-2 which confirmed the diagnosis of COVID-19. The patient was hospitalized in a normal hospital bed. Asymptomatic treatment and an oxygen supply were initiated. The clinical course was marked by the absence of any clinical or biological complications and the patient was discharged 14 days after hospital admission.

\section{DISCUSSION}

There are no specific clinical symptoms which allow COVID-19 to be reliably distinguished from other types of viral respiratory infection. Symptomatic infection ranges from mild to critical, but most infections are not severe. However, some patients with initially mild symptoms may progress to more severe symptoms over the course of a week ${ }^{[2]}$.

Pneumonia evolving to ARDS is the major complication in patients with severe disease and can develop shortly after the onset of dyspnoea. Other reported complications included arrhythmias, acute cardiac injury and shock. Pneumonia is usually accompanied by fever, cough, fatigue, anorexia and myalgia. Of note, fever might not be a universal finding according a Chinese study which reported it could be initially absent or appear during the course of the disease ${ }^{[3]}$. Another study reported lack of respiratory symptoms such as coughing and sputum production but the presence of gastrointestinal symptoms such as nausea, vomiting and diarrhoea in patients positive for COVID-19 [4].

Other Coronaviruses have been associated with anosmia, so it is not surprising that anosmia has been anecdotally reported as a distinguishing symptom in patients ultimately diagnosed with COVID-19. However, available cohort studies have not highlighted this symptom, and its frequency and utility in suspecting COVID-19 is still uncertain ${ }^{[5,6]}$. Anosmia has particularly been seen in patients positive for the Coronavirus with no other clinical features. It is been suggested that the virus causes some sort of inflammation in the olfactory nerves rather than damage to the structure of the receptors.

The olfactory system is designed to survive the constant bombardment of pathogens, so any dysfunction will only be temporary. While the olfactory nerve cells within the nasal lining can be infected and die, stem cells rapidly generate new nerve cells which extend their long connections back up into the brain to restore the pathway. Depending on the severity of the initial infection, the sense of smell can return within a few days or weeks. Patients with taste loss actually have an impaired sense of smell, which has a huge impact on their ability to detect flavour. Smell and taste both contribute to perception of flavour, making difficult for patients to separate the two senses.

\section{CONCLUSIONS}

Anosmia, hyposmia and dysgeusia in the absence of other respiratory diseases such as allergic rhinitis, acute rhinosinusitis or chronic rhinosinusitis, should alert physicians to the possibility of COVID-19 infection and prompt serious consideration of self-isolation and testing of these individuals. In an effort to establish the true significance of these symptoms in the diagnosis and progression of COVID-19, the American Academy of Otolaryngology-Head and Neck Surgery (AAO-HNS) has issued their 'COVID-19 Anosmia Reporting Tool for Clinicians' ${ }^{\text {[5] }}$.

Anosmia and dysgeusia should prompt COVID-19 infection screening even in the absence of classic respiratory symptoms in the present epidemiological context. 


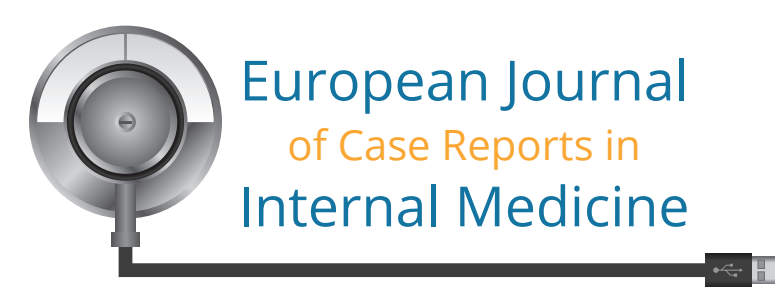

\section{REFERENCES}

1. World Health Organization. Novel coronavirus (2019-nCov): situation report, 2. 22 January 2020. Available from https://apps.who.int/iris/handle/10665/330761 (accessed 2 April 2020).

2. Guan WJ, Ni ZY, Hu Y, et al. Clinical characteristics of coronavirus disease 2019 in China. N Engl J Med 2020 Feb 28. doi: 10.1056/NEJMoa2002032 [Epub ahead of print].

3. Huang C, Wang Y, Li X, et al. Clinical features of patients infected with 2019 novel coronavirus in Wuhan, China. Lancet 2020;395:497.

4. Jin X, Lian J, Hu J, et al. Epidemiological, clinical and virological characteristics of 74 cases of coronavirus-infected disease 2019 (COVID-19) with gastrointestinal symptoms. Gut 2020 Mar 24. pii: gutjnl-2020-320926. doi: 10.1136/gutjnl-2020-320926 [Epub ahead of print].

5. American Academy of Otolaryngology-Head and Neck Surgery. Coronavirus disease 2019: resources. Available from https://www.entnet.org/content/coronavirus-disease2019-resources (accessed 23 March 2020).

6. Giacomelli A, Pezzati L, Conti F, et al. Self-reported olfactory and taste disorders in SARS-CoV-2 patients: a cross-sectional study. Clin Infect Dis 2020 Mar 26. pii: ciaa330. doi: 10.1093/cid/ciaa330 [Epub ahead of print] 